OPEN

SUBJECT AREAS:

DRUG DELIVERY

NANOMEDICINE

Received

11 December 2013

Accepted

6 March 2014

Published

21 March 2014

Correspondence and requests for materials should be addressed to J.-H.C. (jhchoy@ewha. ac.kr) or C.-O.Y. (chaeok@hanyang.ac. kr)

\section{Inorganic Nanovehicle Targets Tumor in an Orthotopic Breast Cancer Model}

\author{
Goeun Choi', Oh-Joon Kwon², Yeonji Oh' ${ }^{1}$, Chae-Ok Yun² \& Jin-Ho Choy
}

${ }^{1}$ Center for Intelligent Nano-Bio Materials (CINBM), Department of Chemistry and Nano Science (Ewha Global Top 5 program-2011) and Department of Bioinspired Science, Ewha Womans University, Seoul 120-750, Republic of Korea, ${ }^{2}$ Department of Bioengineering, College of Engineering, Hanyang University, Seoul 133-791, Republic of Korea.

The clinical efficacy of conventional chemotherapeutic agent, methotrexate (MTX), can be limited by its very short plasma half-life, the drug resistance, and the high dosage required for cancer cell suppression. In this study, a new drug delivery system is proposed to overcome such limitations. To realize such a system, MTX was intercalated into layered double hydroxides (LDHs), inorganic drug delivery vehicle, through a co-precipitation route to produce a MTX-LDH nanohybrid with an average particle size of approximately $130 \mathrm{~nm}$. Biodistribution studies in mice bearing orthotopic human breast tumors revealed that the tumor-to-liver ratio of MTX in the MTX-LDH-treated-group was 6-fold higher than that of MTX-treated-one after drug treatment for $2 \mathrm{hr}$. Moreover, MTX-LDH exhibited superior targeting effect resulting in high antitumor efficacy inducing a $74.3 \%$ reduction in tumor volume compared to MTX alone, and as a consequence, significant survival benefits. Annexin- $\mathrm{V}$ and propidium iodine dual staining and TUNEL analysis showed that MTX-LDH induced a greater degree of apoptosis than free MTX. Taken together, our data demonstrate that a new MTX-LDH nanohybrid exhibits a superior efficacy profile and improved distribution compared to MTX alone and has the potential to enhance therapeutic efficacy via inhibition of tumor proliferation and induction of apoptosis.

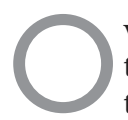

ver past decades, the desire to develop novel rationally designed materials for clinical applications has led to innovative attempts to hybridize diverse constituents that differ from each other at the nanoscale, with the development of nanolevel hybrids between different inorganic materials, organic and inorganic materials, or biomaterials and inorganic materials. Such nanohybridization can result in highly synergistic effects with unusual physico-chemical properties or complementary behaviors between the two hybridized materials ${ }^{1-14}$. In particular, biomaterial-inorganic nanohybrids possess unique properties that make them suitable for biological applications, providing stabilization, targeted delivery, and the controlled release of incorporated drugs or bioactive molecules ${ }^{8,15-19}$.

Layered double hydroxides (LDHs), also known as anionic clays, are promising candidates as drug delivery carriers. LDHs possess positively charged metal hydroxide layers within which solvated exchangeable anions are stabilized through charge neutralization ${ }^{20-23}$. The general formula of LDHs is $\left[\mathrm{M}^{\mathrm{II}}{ }_{1-\mathrm{x}} \mathrm{M}^{\mathrm{III}}{ }_{\mathrm{x}}(\mathrm{OH})_{2}\right]^{\mathrm{x+}}\left(\mathrm{A}^{\mathrm{n}-}\right)_{\mathrm{x} / \mathrm{n}} \cdot \mathrm{yH}_{2} \mathrm{O}$, where $\mathrm{M}^{\mathrm{II}}, \mathrm{M}^{\mathrm{II}}$ are divalent and trivalent metal cations $\left(\mathrm{M}^{\mathrm{II}}=\mathrm{Mg}^{2+}, \mathrm{Zn}^{2+}, \mathrm{Ni}^{2+}, \mathrm{Ca}^{2+}, \mathrm{Cu}^{2+}\right.$ etc.; $\mathrm{M}^{\mathrm{III}}=\mathrm{Al}^{3+}, \mathrm{Fe}^{3+}$ etc.), and $\mathrm{A}^{\mathrm{n}-}$ represents interlayer anions (e.g., $\mathrm{CO}_{3}{ }^{2-}, \mathrm{NO}_{3}{ }^{-}, \mathrm{SO}_{4}{ }^{2-}$, and other anionic species) (Supplementary Figure $\mathrm{S} 1)^{24,25}$. The layer structure of LDHs is constructed with a brucite-like stacking of $\mathrm{Mg}(\mathrm{OH})_{2}$, in which $\mathrm{Mg}(\mathrm{OH})_{6}$ octahedra are connected through edge sharing to form $4.8-\AA$ thick two-dimensional layers. Substitution of some of the divalent cations in the brucite layer with trivalent cations such as $\mathrm{Al}^{3+}$ results in a permanent cationic charge in the layer. The interlayer space is occupied by charge-balancing anions that are typically bound to the layer through electrostatic interaction and hydrogen bonding with water molecules. The exchangeability of these interlayer anions depends on their electrostatic interaction with the positively charged layer but, with the exception of carbonate ions, most organic and inorganic anions are exchangeable. According to our previous study, we found that LDH particles are least toxic compared to the other nanoparticles such as iron oxide, silica, micelles, liposomes, graphene oxide, carbon nanotubes $\operatorname{etc}^{26-28}$. As a result, LDHs are widely applicable to various supramolecular structures or heterogeneous hybrid systems. LDHs are currently attracting increasing attention as potential components of delivery systems for drugs, genes, or biofunctional molecules, a role that critically depends on their interaction with organic materials and biomolecules. In addition, various biofunctional molecule- and drug-loaded LDHs can be stabilized in body fluids during systemic application and exhibit considerably higher cellular uptake than free drugs $s^{24,29-31}$. 

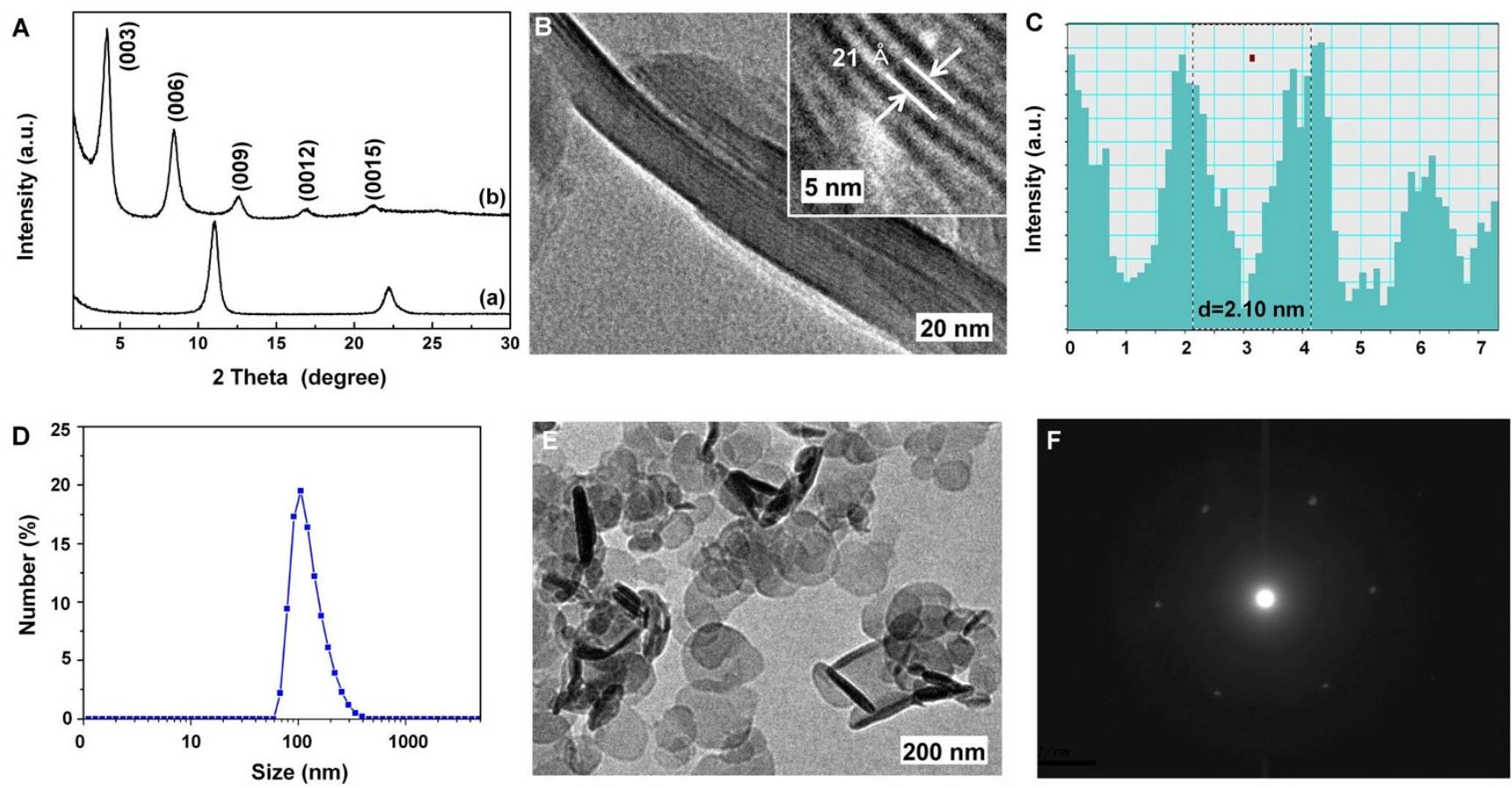

Figure $1 \mid$ (A) X-ray diffraction (XRD) patterns of (a) pure LDH and (b) MTX-LDH, (B) and (C) high-resolution transmission electron microscopy (HR-TEM) images of MTX-LDH nanohybrid: (B) high-resolution micrograph, (C) intensity histogram of the area shown in the inset of (B), (D) particle size distribution of MTX-LDH nanohybrid, (E) high-resolution transmission electron microscopy (HR-TEM) image of MTX-LDH nanohybrid, and (F) represents the selected area electron diffraction (SAED) patterns for MTX-LDH nanohybrid.

In a previous study we prepared nanohybrid molecules for delivery of the chemotherapeutic drug methotrexate (MTX; (2S)-2[(4-\{[(2,4-diamino-7,8-dihydropteridin-6-yl)methyl $]$ (methyl)amino\}phenyl)formamido]pen-tanedioic acid), which has used in the treatment of certain human cancers, including osteosarcoma and leukemia. By functioning as a folate antagonist, MTX inhibits the activation of dihydrofolate reductase (DHFR) in the cell, which catalyzes the reduction of dihydrofolate to tetrahydrofolate in a folate cycle that is coupled with DNA synthesis and cell proliferation. Since this effectively blocks the production of thymidine and de novo DNA synthesis, uptake of MTX into the cytosol ultimately results in cell death ${ }^{29,32-34}$. However, because of its very short plasma half-life and high rate of efflux relative to influx, a high administration dose of MTX is required. To overcome these limitations, we attempted to encapsulate MTX into LDH layers to form hybridized MTX-LDH. Our previous studies confirmed that clathrin-mediated cellular uptake of LDHs results in efficient transportation of drug-loaded LDHs in the size range from 50 to $200 \mathrm{~nm}$ into cells ${ }^{14,20,31}$. In our previous study, the release profiles for simulated intracellular lysosomal condition $(\mathrm{pH}=$ $4.5)$ and simulated body fluid one $(\mathrm{pH}=7.4)$ were evaluated into two steps, a rapid release within $60 \mathrm{~min}$ and a sustained one afterwards as the Bhaskar diffusion model ${ }^{35}$.

In this study, a major novelty has been made for designing injectable device with the 2-dimensional inorganic nanovector in real animal tumor model, orthotopic breast cancer Model, for the first time, in the world. We successfully intercalated MTX into the interlayer space of LDHs through a co-precipitation route. The resulting MTX-LDH was characterized by powder X-ray diffraction (PXRD) and transmission electron microscopy (TEM) analyses. TEM and particle size distribution studies indicated that the MTX-LDH particles showed an even size distribution with an average particle size of approximately $130 \mathrm{~nm}$. The use of nanoparticles with a size of 100 to $200 \mathrm{~nm}$ as delivery systems should result in effective tumor targeting via the enhanced permeation and retention (EPR) effect, by which molecules within certain size ranges (such as nanoparticles and macromolecules) tend to accumulate to a higher level in tumor tissues than in normal tissues ${ }^{36-38}$. In addition, we evaluated the potential liver toxicity of MTX-LDH nanohybrids and free MTX through in vivo experiments. Finally, we compared the therapeutic efficacy of MTX-LDH nanohybrids and free MTX in an orthotopic breast cancer model to determine the efficiency of drug delivery via the nanohybrid system.

\section{Results}

Powder X-ray diffraction analysis. Figure $1(\mathrm{~A})$ shows the PXRD patterns of LDH and MTX-LDH. The chemical compositions of pure $\mathrm{LDH}$ and the MTX-LDH nanohybrid prepared in this study were $\mathrm{Mg}_{0.667} \mathrm{Al}_{0.333}(\mathrm{OH})_{2} \mathrm{Cl}_{0.333} \cdot 0.52 \mathrm{H}_{2} \mathrm{O}$ and $\mathrm{Mg}_{0.655} \mathrm{Al}_{0.344}(\mathrm{OH})_{2}$ $\left[(\mathrm{MTX})_{0.107} \mathrm{Cl}_{0.128}\right] \cdot 0.1 \mathrm{H}_{2} \mathrm{O}$, respectively (Supplementary Table S1). Pure LDH exhibited a series of well-developed (00l) reflections ${ }^{39}$, which were also seen with MTX-LDH. The basal spacing of pure LDH was estimated to be $7.69 \AA$, whereas after intercalation of MTX into the LDH layer the basal spacing clearly increased to 21.0 $\AA$. Given the 4.8 - $\AA$ layer thickness of $\mathrm{LDH}$, the gallery height of the MTX-LDH could be confirmed as $16.2 \AA$. Therefore, considering that the longitudinal and lateral molecular dimensions of MTX are $21.2 \AA$ and $6.5 \AA$, respectively, the MTX molecules appear to be well oriented between the LDH layers with a tilted angle of $43^{\circ 30}$.

High-resolution transmission electron microscopy (HR-TEM) studies of MTX-LDH. Cross-sectional high-resolution transmission electron microscopy (HR-TEM) images of the MTX-LDH nanohybrid revealed a well-ordered layered structure (Fig. 1B, C). The interlayer distance between the periodic lattice fringes from the $(00 l)$ planes in MTX-LDH was approximately $21.0 \AA$, in good agreement with the $\mathrm{d}_{\text {ool }}$ value of $21.0 \AA$ determined from the XRD pattern. The intensity values of the region of the HR-TEM micrograph shown in the inset image of Figure $1 \mathrm{~B}$ are also consistent with these results (Fig. 1C). 


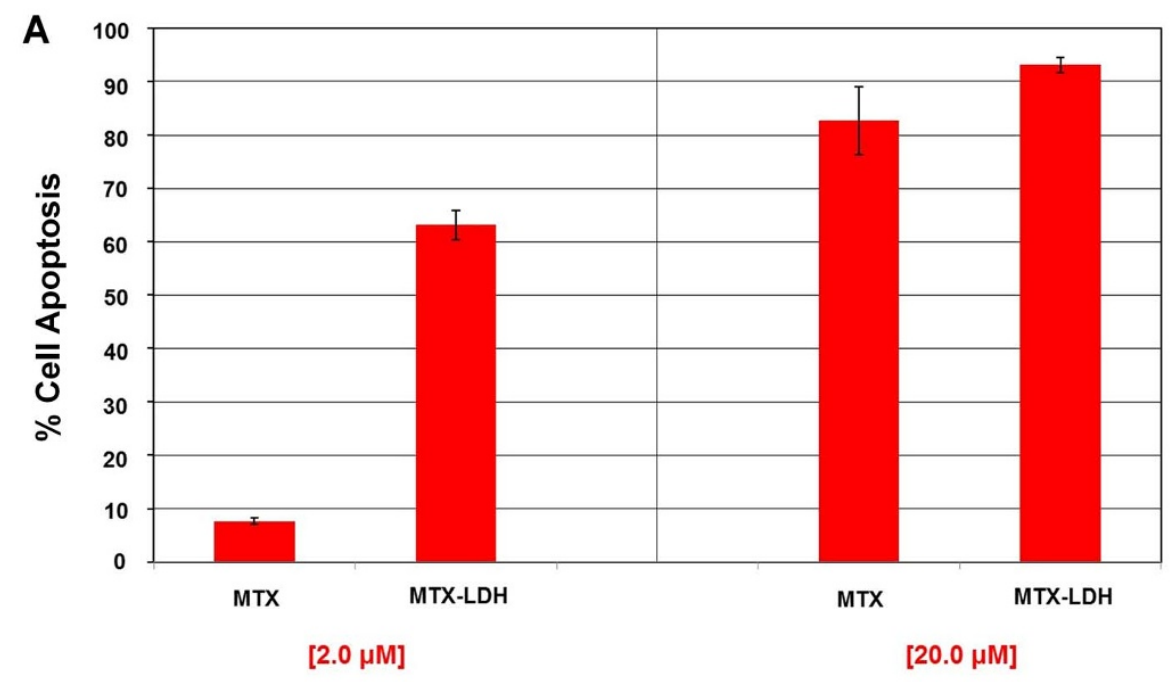

72h post-transfection

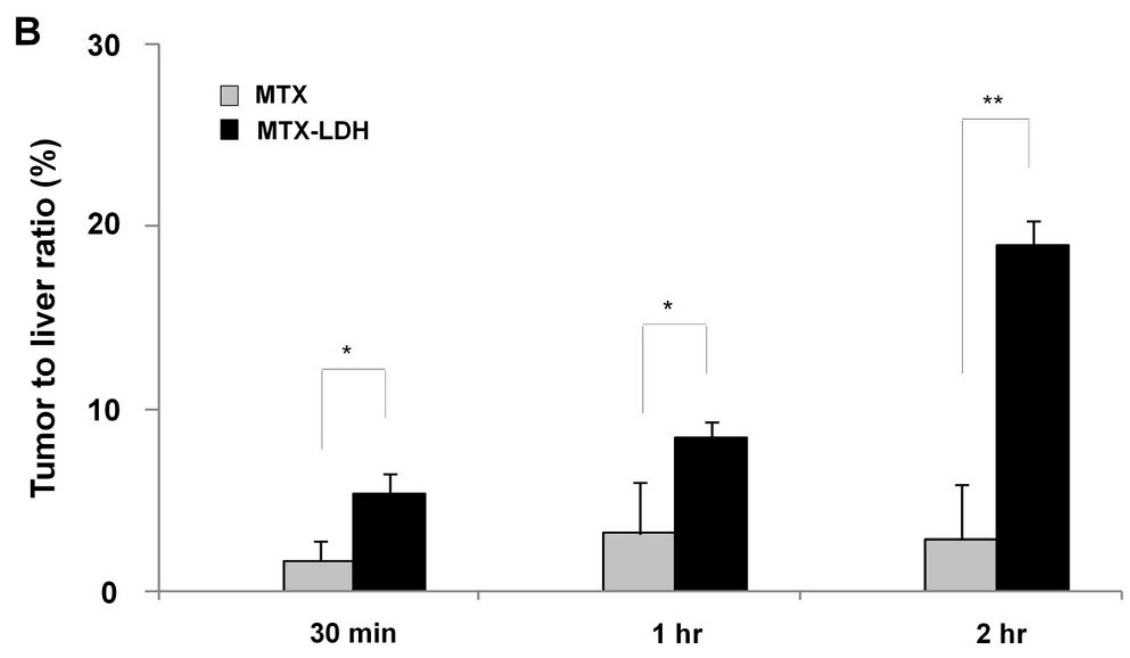

Figure $2 \mid$ (A) Quantification of cell death by flow cytometric analysis of PI and Annexin V staining after 72-hr treatment with MTX or MTX-LDH (Data show mean \pm S.D.), and (B) biodistribution studies for tumor-to-liver ratio of MTX in MTX( $\square$ )- treated group and MTX-LDH ( $\square$ )-one. $\left({ }^{*} \mathrm{p}<0.05,{ }^{* *} \mathrm{p}<0.01\right)$.

Particle size and surface charge of MTX-LDH. Figure 1D,E shows TEM images and particle size distribution of MTX-LDH. The average size of the MTX-LDH nanohybrid particles was approximately $130 \mathrm{~nm}$, in good agreement with the zetasizer results (Fig. 1D). Various long-circulating nanoparticulate pharmaceutical carriers spontaneously accumulate in various pathological sites, such as solid tumors areas, via the enhanced permeability and retention (EPR) effect ${ }^{36-38}$. Similarly, our MTX-LDH nanohybrid drug carriers might penetrate through the leaky pathological vasculature into the tumor interstitium and degrade there, releasing free drug and creating a high local concentration. The surface charges $(\xi$ potential) show that the LDH particles have a positive layer charge due to the isomorphous substitution of $\mathrm{Mg}^{2+}$ ions by $\mathrm{Al}^{3+}$ ions, explaining why the negatively charged MTX molecules could be intercalated into the interlayer space of LDHs thus neutralizing the charge of the reaction (Supplementary Table S1).

Cytotoxicity of the MTX-LDH nanohybrid. Cell death was quantified by flow cytometric analysis of propidium iodide (PI) and Annexin V markers, which stain cells that are dead or undergoing death. Treatment of cultured MCF7/mot cells with MTX or MTX-LDH at a concentration of $20.0 \mu \mathrm{M}$ for $72 \mathrm{hr}$ resulted in death of $82.73 \%$ and $93.10 \%$ of the cells, respectively
(Fig. 2A). However, following 72-hr treatment with $2.0 \mu \mathrm{M}$ MTX or MTX-LDH, the total percentage of dead cells and cells undergoing cell death was $7.65 \%$ for MTX and $63.14 \%$ for MTX-LDH. Therefore at the lower concentration $(2.0 \mu \mathrm{M})$, the MTX-LDH-treated cells exhibited an 8-fold higher level of cell death than the MTX-treated ones. This presumably reflects the enhanced therapeutic effect of a lower dose of MTX-LDH.

Tumor-to-liver ratio of MTX-LDH. We next performed biodistribution studies to determine whether MTX-LDH was efficiently delivered to tumors in vivo. Mice bearing orthotopic MCF-7/mot human breast tumors were treated intraperitoneally with PBS, LDH, MTX, or MTX-LDH. Liver and tumor tissues were harvested at $30 \mathrm{~min}, 1 \mathrm{hr}$, and $2 \mathrm{hr}$ after injection and the level of MTX in each tissue was determined. As shown in Figure 2B, the tumor-to-liver ratio of MTX was $1.7 \%, 3.2 \%$, and $2.9 \%$ at $30 \mathrm{~min}, 1 \mathrm{hr}$, and $2 \mathrm{hr}$ post injection, respectively. In marked contrast, the corresponding tumor-to-liver ratios of MTX in the MTX-LDH-treated group were $5.40 \%, 8.40 \%$, and $19.02 \%$, respectively. This represents a $6-$ fold increase in the tumor-to-liver bioaccumulation ratio for MTXLDH compared with free MTX. Since the tumor-to-liver ratio is a very important indicator of both the therapeutic efficacy and the safety profile, the enhanced tumor-to-liver ratio for MTX-LDH 

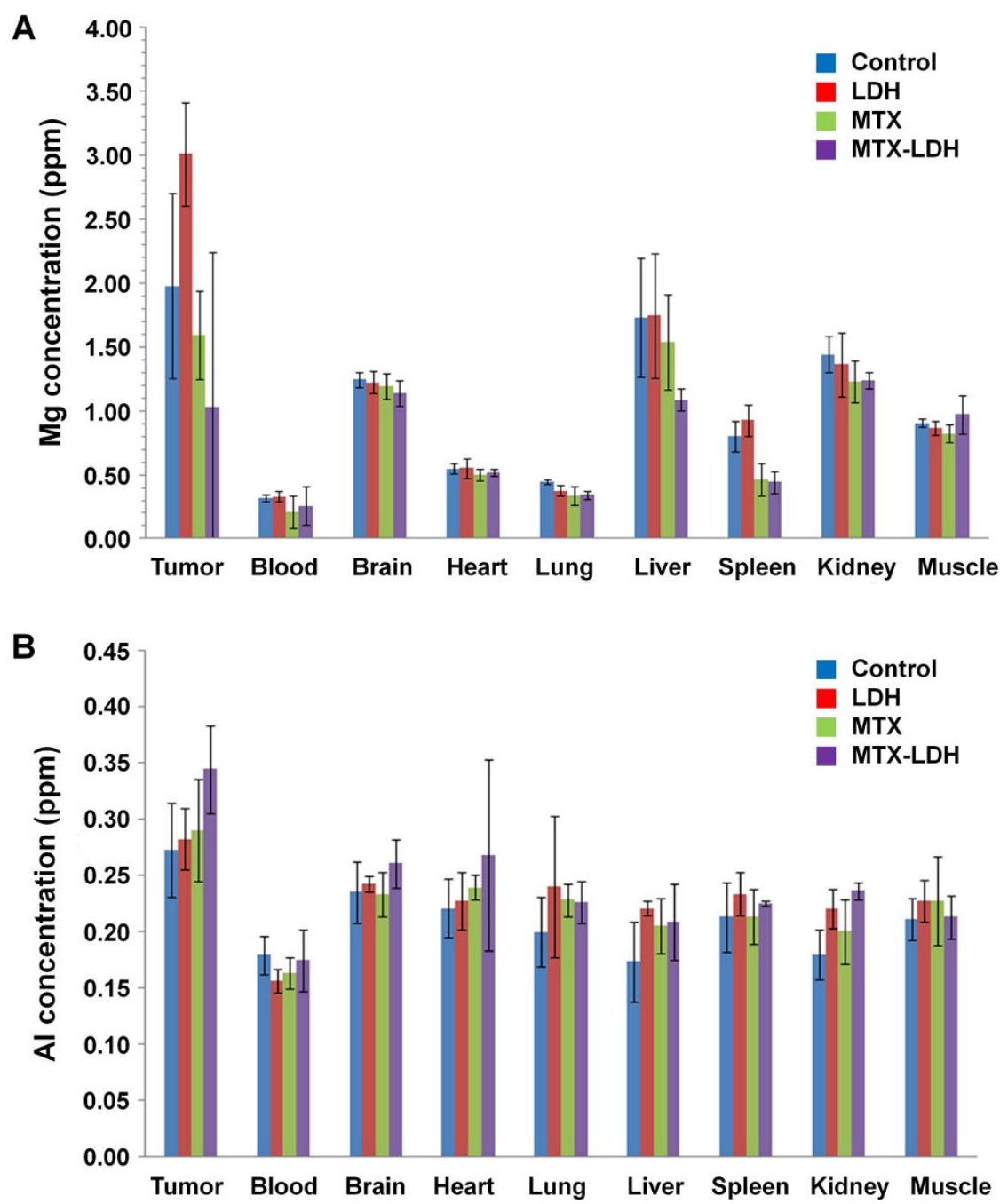

Figure $3 \mid$ (A) and (B) accumulation of LDH in organs of tumor-bearing mice treated with PBS (control, $\square$ blue), LDH ( $\square$ red), MTX ( $\square$ green), or MTX-LDH ( $\square$ violet) for 5 weeks ( $n=3$ for each group). On the first day after the final treatment, the mice were sacrificed and organs, blood, and tumors were collected for measurement of $\mathrm{Mg}$ and $\mathrm{Al}$, the major components of $\mathrm{LDH}$.

indicates its high potential as an effective and safe systemic therapy for the treatment of cancer patients.

Accumulation of LDH. To investigate the accumulation of LDH after treatment, tumors and various organs were harvested from each treatment group 1 day after the last injection and digested. The concentration of $\mathrm{Mg}$ and $\mathrm{Al}$ ions, the major components of $\mathrm{LDH}$, was measured by ICP-AES. As shown in Figures $3 \mathrm{~A}$ and 3B, the levels of $\mathrm{Mg}$ and $\mathrm{Al}$ ions were similar among the groups and within the error range for all organs tested. Interestingly, the $\mathrm{LDH}$ nanovehicles did not appear to significantly accumulate in tissue during treatment of the MCF7/mot orthotopic breast cancer mouse model.

In-vivo anti-tumor activity and survival rates in an orthotopic breast cancer model. To compare the therapeutic efficacy of MTX and MTX-LDH, we performed a tumor growth inhibition test in a MCF7/mot orthotopic breast cancer model. Figure 4 shows tumor volumes after intraperitoneal injection of PBS, LDH, MTX, or MTXLDH into tumor-bearing mice. The mean tumor volumes of the PBSand LDH-treated groups on day 32 were $3374.6 \mathrm{~mm}^{3}$ and $3638.5 \mathrm{~mm}^{3}$, respectively (Fig. 4A). Following administration of MTX, the tumor volume was reduced to $2447.6 \mathrm{~mm}^{3}$, representing a $27.5 \%$ reduction compared with the PBS-treated group. MTX-LDH had an even stronger antitumor effect than MTX with a mean tumor volume of $627.8 \mathrm{~mm}^{3}$, representing $81.4 \%$ and $74.3 \%$ reductions in tumor volume compared with the PBS group and the MTX group, respectively. This inhibitory effect of MTX-LDH on tumor growth was associated with a remarkable increase in survival. On day 32 , only one mouse was surviving among a total of six mice treated with PBS, representing a $16.7 \%$ survival rate (Fig. 4B). The groups treated with LDH or MTX showed survival rates of $33.3 \%$ and $66.6 \%$, respectively. In contrast, all of the mice injected with MTX-LDH survived until day 32 . Taken collectively, these results suggest that MTX-LDH significantly suppresses the growth of orthotopic breast tumors and increases the survival rate compared with free MTX, consistent with the results of the biodistribution study (Fig. 2B).

Increased apoptosis in tumors. The mechanism underlying the enhanced anti-tumor effect and survival benefit of MTX-LDH was further investigated by histological and immunohistochemical analysis. Tumors were harvested from each treatment group 6 days after three sequential treatments. Hematoxylin and eosin (H \& E) staining revealed that the majority of the remaining tumor mass following treatment with MTX-LDH was necrotic, whereas necrotic lesions were only detectable in a limited region of tumors treated with free MTX. To analyze the degree to which MTX-LDH induced apoptosis in vivo we performed a TUNEL assay on tumor 
A

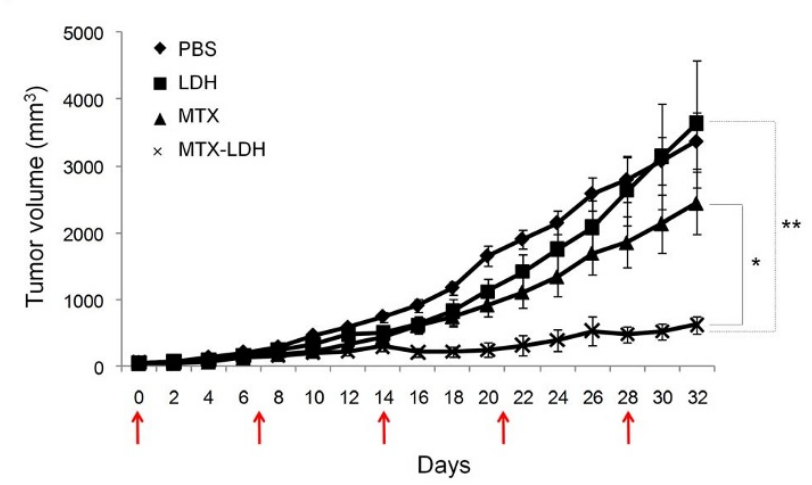

B

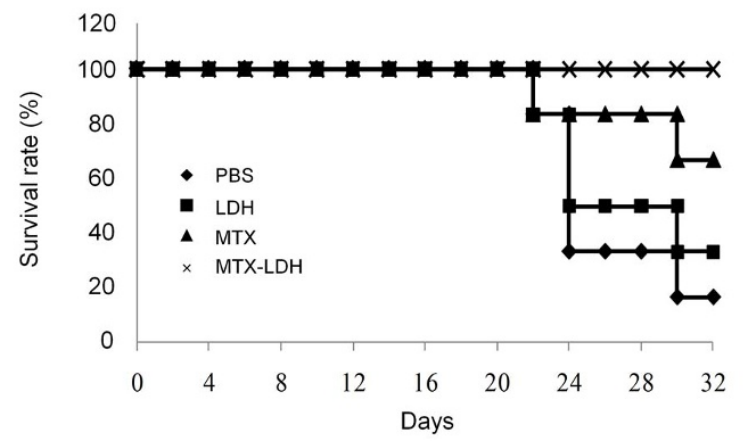

Figure $4 \mid$ (A) Anti-tumor activity of MTX and MTX-LDH in the MCF7/ mot orthotopic breast cancer model. PBS $(\boldsymbol{\bullet}), \operatorname{LDH}(\boldsymbol{\square}), \operatorname{MTX}(\boldsymbol{\Delta})$, and MTX-LDH (X) were administered via intraperitoneal injection on days 0 , $7,14,21$, and 28 (indicated by arrows), and tumor volume was measured using calipers every 2 days. (B) Survival rate of tumor-bearing mice treated as in (A). $(* \mathrm{p}<0.05, * * \mathrm{p}<0.01)$.

sections. Qualitative microscopic examination of TUNEL-stained sections revealed very few positive spots in tumors treated with PBS or LDH (Fig. 5A). In the MTX-treated tumor, TUNELpositive nuclei were marginally detected in an area that overlapped with necrotic lesions by H\&E staining. In contrast, the number of TUNEL-positive apoptotic cells was significantly higher in MTXLDH-treated tumor tissue than in PBS-, LDH-, or MTX-treated tumor tissue. Quantification of the TUNEL-positive spots showed that tumor sections from the groups treated with PBS or LDH contained $0.8 \%$ and $0.6 \%$ positive spots, respectively. The number of TUNEL-positive cells increased to $14.0 \%$ in the group treated with MTX and, more importantly, to $49.6 \%$ in the group treated with MTX-LDH ( $\mathrm{P}<0.001$ versus which group) (Fig. 5B), demonstrating that MTX-LDH induces apoptosis and necrosis in tumor tissue at a much higher level than that observed in the other groups. Consistent with this result, administration of MTX-LDH strongly reduced the degree of staining with antibody against PCNA, a marker of proliferating cells that is associated with the $S$ phase of DNA replication ${ }^{40}$, whereas intense staining and a high number of PCNA-positive cells were observed in the groups treated with PBS, LDH, or MTX. Together, these findings suggest that the enhanced antitumor effect of MTX-LDH resulted from inhibition of cell proliferation and promotion of apoptosis in the tumor.

In-vivo safety profiles of MTX-LDH treatment. To investigate potential liver toxicity, serum levels of alanine aminotransferase (ALT) and aspartate aminotransferase (AST) were analyzed at 3 days after intraperitoneal administration of PBS, LDH, MTX, or MTX-LDH. As shown in Figure 6A, none of the groups showed a

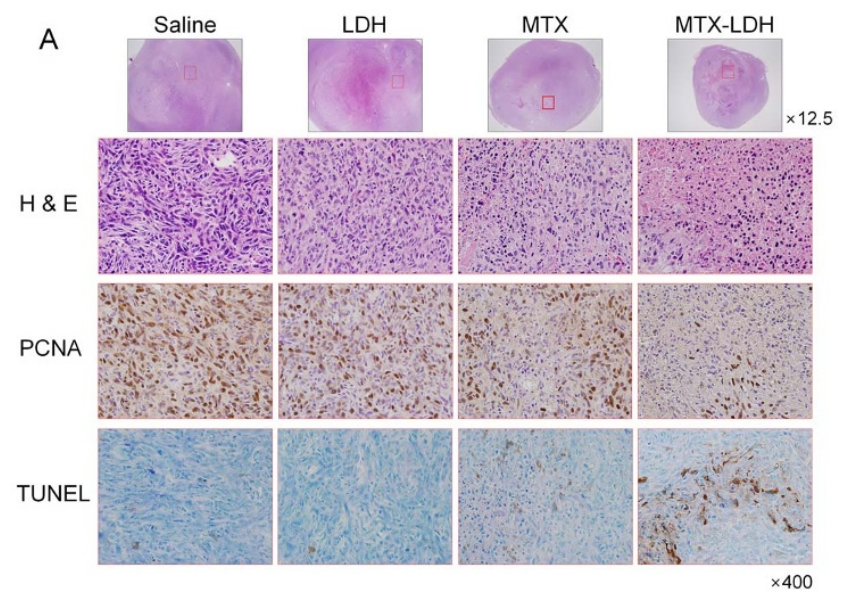

B

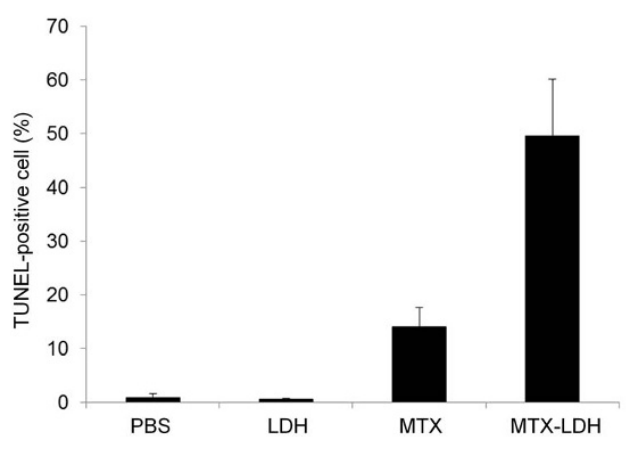

Figure 5 | (A) Histological and immunohistological analysis of tumor tissue sections from MCF-7/mot orthotopic tumor-bearing mice. Representative sections were stained with hematoxylin and eosin ( $\mathrm{H} \& \mathrm{E})$, with antibody specific for proliferating cellular nuclear antigen (PCNA), and by TUNEL staining for apoptotic cells. Original magnification: $\times 12.5$, $\times 400$. (B) Quantification of TUNEL-positive spots in tumor sections of PBS, LDH, MTX, and MTX-LDH-treated groups.

significant increase in ALT and AST levels, which were within the normal range for all treatments (AST: 37-329U/L, ALT: 7-227 U/L). Furthermore, histopathologic analysis of hematoxylin-eosin stained liver sections demonstrated no morphological abnormalities related to liver injury in any of the treatment groups (Fig. 6B). Taken together, these results indicate that systemic delivery of MTX-LDH does not induce liver toxicity.

\section{Discussion}

We have successfully developed an MTX-LDH nanohybrid system with an average particle size of approximately $130 \mathrm{~nm}$ for efficient drug delivery to tumors. In biodistribution studies, mice treated with MTX-LDH had a 6-fold higher tumor-to-liver ratio of MTX than those treated with free MTX. In addition, the MTX-LDH nanohybrid exhibited higher drug efficacy than free MTX in a tumor growth inhibition test using an orthotopic breast cancer mouse model. In vivo safety profiles indicated that incorporation of MTX into LDH nanoparticles provides a non-toxic carrier system for drug delivery to tumors. The homogeneous particle size, biocompatibility, and high therapeutic value of the MTX-LDH nanohybrid system make it a promising injectable nanomedicine for the treatment of cancer.

\section{Methods}

Materials. MTX $\left(\mathrm{C}_{20} \mathrm{H}_{22} \mathrm{~N}_{8} \mathrm{O}_{5}\right.$, purity $\left.>98.0 \%\right)$, magnesium chloride hexahydrate $\left(\mathrm{MgCl}_{2} \cdot 6 \mathrm{H}_{2} \mathrm{O}\right.$, purity $>98.0 \%$, pharmacopoeia grade), and aluminum chloride hexahydrate $\left(\mathrm{AlCl}_{3} \cdot 6 \mathrm{H}_{2} \mathrm{O}\right.$, purity $>99.0 \%$, pharmacopoeia grade) were purchased from Sigma-Aldrich Co. Ltd. (Korea). 

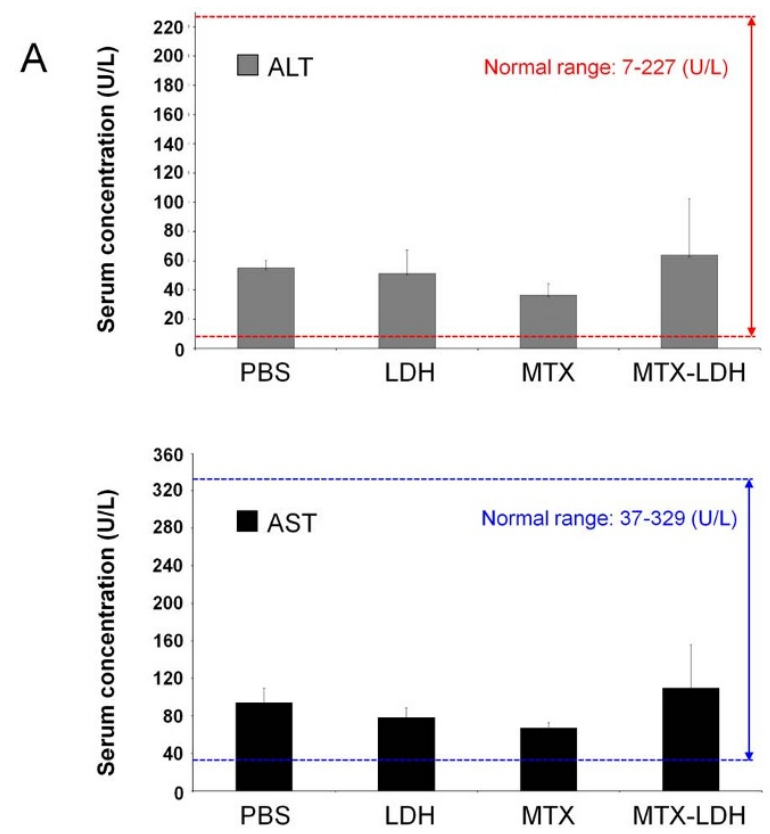

B

PBS

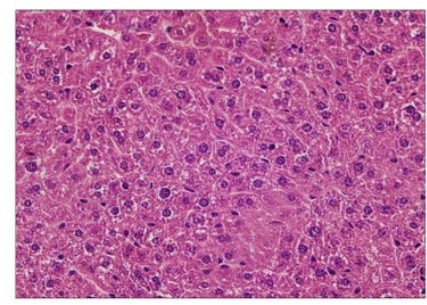

MTX

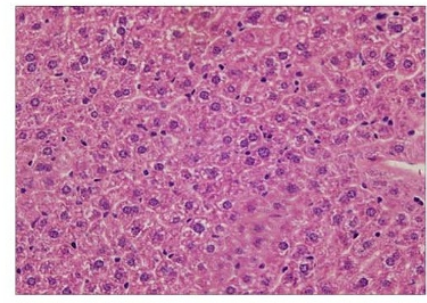

$\mathrm{LDH}$

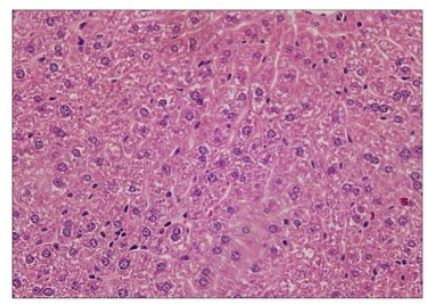

MTX-LDH

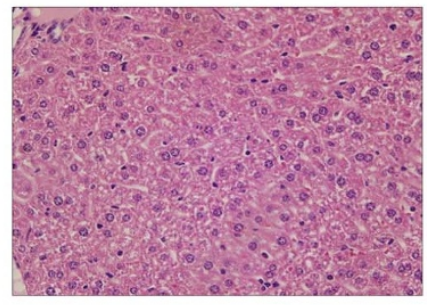

Figure 6 Liver toxicity studies to assess liver damage and enzyme function after drug treatment. (A) Aspartate aminotransferase (AST) and alanine aminotransferase (ALT) levels. (B) Hematoxylin and eosin (H \& E) staining of liver tissues.

Animals. During the experiments, all mice were kept in a laminar air flow cabinet under specific pathogen free conditions at Yonsei University, an Association for Assessment and Accreditation of Laboratory Animal Care-accredited animal facility. All animal experiments were conducted under the institutional guidelines established by the Animal Research Committee. The animal protocol was approved by the Committee on the Ethics of Animal Experiments of the Yonsei University.

Preparation of MTX-LDH nanohybrid. LDH with the formula $\mathrm{Mg}_{0.667} \mathrm{Al}_{0.333}(\mathrm{OH})_{2} \mathrm{Cl}_{0.333} \cdot 0.52 \mathrm{H}_{2} \mathrm{O}$ was prepared by the direct co-precipitation method at room temperature. An aqueous solution of $\mathrm{MgCl}_{2} \cdot 6 \mathrm{H}_{2} \mathrm{O}(0.066 \mathrm{M})$ and $\mathrm{AlCl}_{3} \cdot 6 \mathrm{H}_{2} \mathrm{O}(0.033 \mathrm{M})$ was titrated dropwise with $0.5 \mathrm{M} \mathrm{NaOH}$ solution under a nitrogen atmosphere. The final $\mathrm{pH}$ of the solution was adjusted to $9.5 \pm 0.2$. The resulting white precipitate was aged for $24 \mathrm{hr}$, collected by centrifugation, washed with decarbonated water, and then dried in a vacuum. A MTX-LDH nanohybrid was prepared by the direct co-precipitation method. MTX was dispersed in deionized water and titrated with a $0.5 \mathrm{M} \mathrm{NaOH}$ solution to give a $0.033 \mathrm{M}$ solution of MTX at $\mathrm{pH} 7.0 \pm 0.2$. An aqueous solution of $\mathrm{MgCl}_{2} \cdot 6 \mathrm{H}_{2} \mathrm{O}(0.066 \mathrm{M})$ and $\mathrm{AlCl}_{3} \cdot 6 \mathrm{H}_{2} \mathrm{O}$ $(0.033 \mathrm{M})$ was added to the MTX solution, while maintaining the $\mathrm{pH}$ at $9.5 \pm 0.2$ by simultaneous dropwise addition of $0.5 \mathrm{M} \mathrm{NaOH}$. The resulting slurry was further aged for $48 \mathrm{hr}$ to reach equilibrium. The entire process was performed under a nitrogen atmosphere at room temperature. The resulting yellow precipitate was collected by centrifugation, washed with decarbonated water, and dried in a vacuum.

Sample characterization. For structural analysis, powder X-ray diffraction (PXRD) patterns were obtained using a diffractometer (Rigaku D/MAX RINT 2200-Ultima+, Japan) with monochromatized and Ni-filtered $\mathrm{Cu} \mathrm{K}_{\alpha}$ radiation $(\lambda=1.5418 \AA)$. The diffractometer was operated at $40 \mathrm{kV}$ and $30 \mathrm{~mA}$. The chemical composition of the hybrids was determined using three techniques: elemental CHNS analysis (EA1112, $\mathrm{CE}$ instrument, Italy), inductively coupled plasma-atomic emission spectrometry (ICP-AES) (Optima-4300 DV, PerkinElmer, USA), and high-resolution transmission electron microscopy (HR-TEM), which was carried out on a JEOL JEM-2100F microscope (Japan) operated at an accelerating voltage of $200 \mathrm{kV}$.

Cell culture. The MCF7/mot cell line was received from Dr. Renu Wadhwa (National Institute of Advanced Industrial Science \& Technology, Japan) and cultured in Dulbecco's modified Eagle's medium (Hyclone Lab. Inc., Grand Island, NY) supplemented with $10 \%$ fetal bovine serum (Hyclone), penicillin (100 IU/ml), and streptomycin $(50 \mu \mathrm{g} / \mathrm{ml})$ at $37^{\circ} \mathrm{C}$ in a humidified incubator at $5 \% \mathrm{CO}_{2}$.

Annexin-V and PI dual staining. MCF7/mot cells were seeded in a 12 -well plate at 5 $\times 10^{4}$ cells per well. After culturing for $24 \mathrm{hr}$, cells were treated with MTX-LDH at concentrations of 2.0 and $20.0 \mu \mathrm{M}$ for $72 \mathrm{hr}$ and then stained with PI and Annexin V according to the manufacturer's protocol (BD Pharmingen, San Diego, CA). PIpositive and Annexin V-positive staining was quantified with BD CellQuest ${ }^{\mathrm{TM}}$ Pro software (BD Bioscience, Sparks, MD) using a FACS-Calibur flow cytometer (BD Biosciences, San Jose, CA). Three independent experiments were performed in triplicate and yielded consistent results.
Biodistribution studies. To compare the biodistribution of free MTX and MTX $\mathrm{LDH}, 2 \times 10^{6} \mathrm{MCF} 7 / \mathrm{mot}$ cells were injected into the mammary pad of 7-week-old female athymic nu/nu mice (Orient bio, Korea). When the tumor volume reached approximately $300 \mathrm{~mm}^{3}$, PBS, LDH, MTX (60 mg/kg), or MTX-LDH (containing $60 \mathrm{mg} / \mathrm{kg}$ MTX) ( $\mathrm{n}=3$ per group) was injected intraperitoneally (i.p.), and the mice were sacrificed immediately after $30 \mathrm{~min}, 1 \mathrm{hr}$, and $2 \mathrm{hrs}$. And the tumor and liver were harvested, homogenized, and dissolved in nitric acid. Two volumes of acetonitrile were added to the ground organ and mixed vigorously to precipitate proteins. After centrifugation at $10000 \times g$ for $3 \mathrm{~min}$, four volumes of chloroform were added to the supernatant to remove lipids. The supernatants were collected by centrifugation at $2000 \times g$ for $10 \mathrm{~min}$ and analyzed by high-performance liquid chromatography (HPLC; Agilent 1100 series Instrument, USA). The MTX content in the cells was quantified by HPLC with an Agilent 1100 series HPLC system using a C18 column (Nucleosil, $250 \times 4.6 \mathrm{~mm}$, Alltech, Nicholasville, KY). The mobile phase was $0.05 \mathrm{M} \mathrm{KH}_{2} \mathrm{PO}_{4}, 10 \%$ acetonitrile, and $\mathrm{pH} 6.6$, and the flow rate was $1 \mathrm{~mL} / \mathrm{min}$, with a column temperature of $40^{\circ} \mathrm{C}$. MTX was detected by the UV absorption band at $304 \mathrm{~nm}$.

Accumulation of LDH. To measure the accumulation of $\mathrm{Mg}$ and $\mathrm{Al}$ ions in organs, due to the dissolution of $\mathrm{LDH}$, the tumor-bearing mice were injected intraperitoneally (i.p.) with PBS, LDH, MTX $(10 \mathrm{mg} / \mathrm{kg})$, or MTX-LDH $(10 \mathrm{mg} / \mathrm{kg}$ MTX) once a week for 5 weeks ( $n=3$ per group). And the mice were sacrificed on the first day after the final treatment, and the organs, blood, and tumor tissues were collected. To remove the organic ligands and obtain the metal atoms in solution, each of the tissues was digested in a combined acid containing $\mathrm{HCl}$ and $\mathrm{H}_{2} \mathrm{O}_{2}$ diluted with $\mathrm{DI}$ water. The concentrations of $\mathrm{Mg}$ and $\mathrm{Al}$ ions were analyzed by inductively coupled plasma-atomic emission spectrometry (ICP-AES) (Optima-4300 DV; PerkinElmer, Waltham, MA).

Antitumor activity in an orthotopic breast cancer model. We compared the antitumor efficacy of MTX and MTX-LDH in mice bearing orthotopic MCF7/mot tumors. When the tumor volume reached approximately $60 \mathrm{~mm}^{3}$, the mice were randomly assigned into four groups $(\mathrm{n}=6$ per group) and injected i.p. with PBS, LDH, MTX (10 mg/kg), or MTX-LDH (10 mg/kg MTX) once a week for 5 weeks. Tumor size was measured using calipers every 2 days and the tumor volume was calculated $\left(\right.$ Volume $=$ Length $\times$ Width $\left.^{2} \times 0.523\right)$. The percentage of mice surviving was determined by monitoring events related to tumor growth (tumor size $>$ $\left.2500 \mathrm{~mm}^{3}\right)$.

Histologic and immunohistochemical evaluation of tumors. PBS, LDH, MTX, or MTX-LDH was systemically administered to mice bearing MCF-7/mot orthotopic tumors. Six days after the third treatment, representative tumor tissue was collected from each group, fixed in 10\% formalin, embedded in paraffin (Wax-it, Vancouver, Canada), and cut into 5- $\mu \mathrm{m}$ thick sections. Representative sections were stained with hematoxylin, eosin, and with an antibody specific for proliferating cellular nuclear antigen (M0879, Dakocytomation, Carpinteria, CA). Apoptotic cells were identified by the terminal deoxynucleotidyl transferase deoxyuridine triphosphate nick end 
labeling (TUNEL) assay using an in situ ApopTag kit (Chemicon International, Temecula, CA).

Measurement of inflammatory cytokines and liver toxicity studies. To analyze the innate immune response and liver toxicity after drug treatment, $\mathrm{PBS}(\mathrm{n}=2), \mathrm{LDH}(\mathrm{n}$ $=4)$, MTX (10 mg/kg, $\mathrm{n}=4)$, or MTX-LDH $(10 \mathrm{mg} / \mathrm{kg}$ MTX, $\mathrm{n}=4)$ was intraperitoneally administrated to 6 -week-old balb/c mice. Liver damage was assessed by histologic characteristics and measurement of liver enzymes. At 3 days posttreatment, serum was collected for determination of aspartate aminotransferase (AST) and alanine aminotransferase (ALT) (BIOTOXTECH Corporation, Ochang, Chungcheong, Korea). Liver tissues were fixed in $10 \%$ formalin, sectioned, and stained with Meyer's hemotoxyln and eosin.

1. Choy, J. H., Kwon, S. J. \& Park, G. S. High- $\mathrm{T}_{\mathrm{c}}$ superconductors in the twodimensional limit: $\left[\left(\mathrm{Py}_{-} \mathrm{C}_{\mathrm{n}} \mathrm{H}_{2 \mathrm{n}+1}\right){ }_{2} \mathrm{HgI}_{4}\right]-\mathrm{Bi}_{2} \mathrm{Sr}_{2} \mathrm{Ca}_{\mathrm{m}-1} \mathrm{Cu}_{\mathrm{m}} \mathrm{O}_{\mathrm{y}}(\mathrm{m}=1$ and 2). Science 280, 1589-1592 (1998).

2. $\mathrm{Xu}, \mathrm{Z}$. P. et al. Stable suspension of layered double hydroxide nanoparticles in aqueous solution. J. Am. Chem. Soc. 128, 36-37 (2006).

3. Xu, Z. P. et al. Subcellular compartment targeting of layered double hydroxide nanoparticles. J. Control. Release 130, 86-94 (2008).

4. Wong, Y. Y. et al. Efficient delivery of siRNA to cortical neurons using layered double hydroxide nanoparticles. Biomaterials 31, 8770-8779 (2010).

5. Bao, H. F. et al. Synthesis of well-dispersed layered double hydroxide core@ ordered mesoporous silica shell nanostructure (LDH@mSiO2) and its application in drug delivery. Nanoscale 3, 4069-4073 (2011).

6. Yang, J. H. et al. New inorganic-based drug delivery system of indole-3-acetic acid-layered metal hydroxide nanohybrids with controlled release rate. Chem. Mater. 19, 2679-2685 (2007).

7. Ogawa, M. \& Asai, S. Hydrothermal synthesis of layered double hydroxidedeoxycholate intercalation compounds. Chem. Mater. 12, 3253-3255 (2000)

8. Choy, J. H., Kwak, S. Y., Jeong, Y. J. \& Park, J. S. Inorganic layered double hydroxide as a non-viral vectors. Angew. Chem. Int. Ed. 39, 4042-4045 (2000).

9. Ide, Y., Ochi, N. \& Ogawa, M. Effective and selective adsorption of $\mathrm{Zn}^{2+}$ from seawater on a layered silicate. Angew. Chem. Int. Ed. 123, 680-682 (2011).

10. Maneeratana, V. et al. Fractal inorganic-organic interfaces in hybrid membranes for efficient proton transport. Adv. Funct. Mater. 23, 2872-2880 (2013).

11. Marquez, A. G. et al. Green scalable aerosol synthesis of porous metal-organic frameworks. Chem. Commun. 49, 3848-3850 (2013).

12. Chung, I. W. et al. Evidence of two-dimensional superconductivity in the single crystalline nanohybrid of organic-bismuth cuprate. J. Phys. Chem. B 110, 16197-16200 (2006).

13. Paek, S. M. et al. Exfoliation and reassembling route to mesoporous titania nanohybrids. Chem. Mater. 18, 1134-1140 (2006).

14. Oh, J. M., Choi, S. J., Kim, S. T. \& Choy, J. H. Cellular uptake mechanism of an inorganic nanovehicle and its drug conjugates: enhanced efficacy due to clathrinmediated endocytosis. Bioconjugate Chem. 17, 1411-1417 (2006).

15. Khan, A. I., Lei, L., Norquist, A. J. \& O'Hare, D. Intercalation and controlled release of pharmaceutically active compounds from a layered double hydroxide. Chem. Commun. 2342-2343 (2001).

16. Choi, G. et al. Inorganic-polymer nanohybrid carrier for delivery of a poorlysoluble drug, ursodeoxycholic acid. Int. J. Pharm. 402, 117-122 (2010).

17. Wang, Q. \& O'Hare, D. Recent advances in the synthesis and application of layered double hydroxide (LDH) nanosheets. Chem. Rev. 112, 4124-4155 (2012).

18. Li, S. D. et al. Cellular uptake and gene delivery using layered double hydroxide nanoparticles. J. Mater. Chem. B 1, 61-68 (2013).

19. Gao, X. et al. Intercalation and controlled release properties of vitamin C intercalated layered double hydroxide. J. Solid State Chem. 203, 174-180 (2013).

20. Oh, J. M., Choi, S. J., Lee, G. E., Kim, J. E. \& Choy, J. H. Inorganic metal hydroxide nanoparticles for targeted cellular uptake through clathrin-mediated endocytosis. Chem. Asian J. 4, 67-73 (2009).

21. Hussein, M. Z., Zainal, Z., Yahaya, A. H. \& Foo, D. W. V. Controlled release of a plant growth regulator, $\alpha$-naphthaleneacetate from the lamella of $\mathrm{Zn}$-Al-layered double hydroxide nanocomposite. J. Control. Release 82, 417-427 (2002).

22. Choy, J. H., Oh, J. M., Park, M., Sohn, K. M. \& Kim, J. W. Inorganic-biomolecular hybrid nanomaterials as a genetic molecular code system. Adv. Mater. 16, 1181-1184 (2004).

23. Kim, M. H., Park, D. H., Yang, J. H., Choy, Y. B. \& Choy, J. H. Drug-inorganicpolymer nanohybrid for transdermal delivery. Int. J. Pharm. 444, 120-127 (2013).

24. Choy, J. H., Kwak, S. Y., Park, J. S., Jeong, Y. J. \& Portier, J. Intercalative nanohybrids of nucleoside monophosphates and DNA in layered metal hydroxide. J. Am. Chem. Soc. 121, 1399-1400 (1999).
25. Kwak, S. Y., Waltraud, M. K., Matthew, A. W. \& Choy, J. H. Inorganic delivery vector for intravenous injection. Biomaterials 25, 5995-6001 (2004).

26. Choi, S. J., Oh, J. M. \& Choy, J. H. Human-related application and nanotoxicology of inorganic particles: complementary aspects. J. Mater. Chem. 18, 615-620 (2008).

27. Yokoyama, M. et al. Toxicity and antitumor activity against solid Ttumors of micelle-forming polymeric anticancer drug and its extremely long circulation in blood. Cancer Res. 51, 3229-3236 (1991).

28. Parnham, M. J. \& Wetzig, H. Toxicity screening of liposomes. Chem Phys Lipids. 64, 263-274 (1993)

29. Oh, J. M. et al. Efficient delivery of anticancer drug MTX through MTX-LDH nanohybrid system. J. Phys. Chem. Solids 67, 1024-1027 (2006).

30. Choy, J. H. et al. Layered double hydroxide as an efficient drug reservoir for folate derivatives. Biomaterials 25, 3059-3064 (2004).

31. Choi, S. J. \& Choy, J. H. Layered double hydroxide nanoparticles as target-specific delivery carriers: uptake mechanism and toxicity. Nanomedicine 6, 803-814 (2011).

32. Gilman, A. G., Goodman, L. S. \& Gilman, A. [Chemotherapy of neoplastic diseases] [Brunton, L. L. (ed.)] [1315-1402] (Macmillan, New York, 2006).

33. Heijden, J. W., Dijkmans, B. A. C., Scheper, R. J. \& Jansen, G. Drug Insight: resistance to methotrexate and other disease-modifying antirheumatic drugsfrom bench to bedside. Nat. Clin. Pract. Rheum. 3, 26-34 (2007).

34. McGuire, J. J. Anticancer antifolates: Current status and future directions. Curr. Pharm. Des. 9, 2593-2613 (2003).

35. Kim, J. Y., Choi, S. J., Oh, J. M., Park, T. \& Choy, J. H. Anticancer drug-inorganic nanohybrid and its cellular interaction. J. Nanosci. Nanotechnol. 7, 3700-3705 (2007).

36. Torchilin, V. P. Targeted Pharmaceutical Nanocarriers for Cancer Therapy and Imaging. The AAPS Journal 9, E128-E147 (2007).

37. Maeda, H. SMANCS and polymer-conjugated macromolecular drugs: advantages in cancer chemotherapy. Adv. Drug Deliv. Rev. 46, 169-185 (2001).

38. Maeda, H., Sawa, T. \& Konno, T. Mechanism of tumor-targeted delivery of macromolecular drugs, including the EPR effect in solid tumor and clinical overview of the prototype polymeric drug SMANCS. J. Control. Release 74, 47-61 (2001).

39. Kloprogge, J. T. \& Frost, R. L. Fourier Transform Infrared and Raman Spectroscopic Study of the Local Structure of $\mathrm{Mg}-$, $\mathrm{Ni}-$-, and $\mathrm{Co}-\mathrm{Hydrotalcites.}$ J. Solid State Chem. 146, 506-515 (1999).

40. Mancini, R., Marucci, L., Benedetti, A., Jezequel, A. M. \& Orlandi, F. Immunohistochemical analysis of S-phase cells in normal human and rat liver by PC10 monoclonal antibody. Liver 14, 57-64 (1994).

\section{Acknowledgments}

We thank Jaeyong Cho for his help in cytofluorometry studies. This work was supported by a National Research Foundation of Korea (NRF) Grant funded by the Korean Government (MSIP) (2005-0049412, J.-H. Choy) and (2010-0029220, C.-O. Yun), and partly by Hyundai IBT Co., Ltd. G. Choi is thankful to the Solvay Scholarship.

\section{Author contributions}

G.C. and O.-J.K. carried out the experiments, analyzed the data, and wrote the manuscript. Y.O. assisted experiments and manuscript. C.-O.Y. contributed to critical discussions on in-vivo studies. J.-H.C. conceived the original idea, designed and supervised the whole study, and made critical comments on the manuscript.

\section{Additional information}

Supplementary information accompanies this paper at http://www.nature.com/ scientificreports

Competing financial interests: The authors declare no competing financial interests.

How to cite this article: Choi, G., Kwon, O.-J., Oh, Y., Yun, C.-O. \& Choy, J.-H. Inorganic Nanovehicle Targets Tumor in an Orthotopic Breast Cancer Model. Sci. Rep. 4, 4430; DOI:10.1038/srep04430 (2014)

his work is licensed under a Creative Commons Attribution-

NonCommercial-NoDerivs 3.0 Unported license. To view a copy of this license visit http://creativecommons.org/licenses/by-nc-nd/3.0 